\title{
Mineração
}

\section{Otimização econômica de explotações a céu aberto}

\author{
Frederico Augusto Rosa do Carmo \\ MSc., Bolsista da CAPES.E-mail: frederico.carmo@cvrd.com.br
}

Adilson Curi

Prof., Dr., Programa de Pós-Graduação em Engenharia Mineral. Departamento de Engenharia de Minas Escola de Minas da Universidade Federal de Ouro Preto. E-mail: curi@demin.ufop.br

Wilson Trigueiro de Sousa

Prof., Dr., Programa de Pós-Graduação em Engenharia Mineral. Departamento de Engenharia de Minas Escola de Minas da Universidade Federal de Ouro Preto. E-mail: trigueiro@demin.em.ufop.br

\section{Resumo}

A determinação dos limites da cava final de qualquer projeto de mineração é um dos maiores desafios de um projeto de mineração. Tais limites precisam ser definidos já no início dos trabalhos de planejamento de lavra e devem ser reconsiderados, novamente e rotineiramente, durante toda a vida útil da mina. Um dos propósitos do plano de exaustão de minas a céu aberto é determinar a cava final ótima, baseando-se em um modelo econômico sujeito a restrições técnicas e visando à maximização do valor atual líquido do projeto. O primeiro algoritmo específico relacionado ao tema foi publicado por Lerchs e Grossmann (1965). O algoritmo de Lerchs e Grossmann tem sido aceito como padrão de teste em comparação com outros algoritmos equivalentes, que têm sido propostos, porque ele resolve o problema correspondente, segundo a teoria dos grafos, ao corte mínimo e fluxo máximo. Outros algoritmos de otimização de cavas finais de minas a céu aberto também são apresentados nesse trabalho. Além disto, um programa acadêmico para a otimização de cavas foi desenvolvido pelos autores sendo que os fundamentos deste são apresentados no presente artigo.

Palavras-chaves: planejamento, lavra, otimização, cava.

\begin{abstract}
The determination of the final pit limits of any mining project is one of the most important challenges in a mining project. Such limits need to be defined at the beginning of the mine planning and should be reconsidered, again and routinely, during all the useful life of a mine. One of the purposes of the open pit mine exhaustion plan is the determination of the optimal final pit, based on an economic model subject to technical restrictions and aimed to maximize the net present value. LERCHS e GROSSMANN (1965) published the first specific algorithm related to the theme. This algorithm has been accepted as standard in comparison to other equivalent algorithms which have been proposed, because it solves the corresponding problem, according to the graph theory, of the minimum cut and maximum flow. Other final pit optimization algorithms are presented in this study too. The authors developed an academic program for pit optimization and the principles of this program are presented in this article.
\end{abstract}

Keywords: planning, mining, optimization, open pit. 


\section{Introdução}

Os limites finais da cava definem o tamanho e a forma de uma mina a céu aberto no final de sua vida útil, buscando a maximização da riqueza futura. Esses contornos finais definem a extensão da reserva lavrável e a quantidade de material estéril a ser retirado. Normalmente, marcam a fronteira-limite, além da qual a explotação não será mais econômica. Os limites finais da cava delimitam uma fronteira, dentro da qual as estruturas de superfície da mina, tais como plantas de beneficiamento e escritórios, não devem ser posicionadas.

\section{Metodologia de cálculos}

\subsection{Apresentação do problema}

Até a década de setenta, a otimização de cavas finais, na mineração, era feita pelo método manual, de tentativas. Segundo tal método, procurava-se chegar a uma cava que fosse econômica e, caso houvesse estéril dentro dessa cava além do admissível, a mesma deveria ser redesenhada, para se obter, se possível, mais minério e menos estéril. Na medida do possível, a cava deveria ser ampliada “em nova(s) tentativa(s) e, assim, sucessivamente, até se alcançar uma cava final satisfatória” (Valente, 1982).

Lerchs e Grossmann (1965) apresentaram um algoritmo matemático que permite "projetar o contorno de uma explotação a céu aberto de tal forma que se maximize a diferença entre o valor total do minério explotado e o custo total da extração do minério e do estéril’. A metodologia de cálculo desse algoritmo tem sido explicada através da teoria matemática dos grafos.

Matematicamente, o problema da cava ótima pode ser representado por um grafo $G=(V, A)$, definido como a reunião de um conjunto $\mathrm{V}$ de elementos $\operatorname{Vj}(j=1,2, \ldots, n)$ chamados de nós de $\mathrm{G}$, com um conjunto A de pares de elementos de $x$, chamados de arcos ou ramos do grafo.
A cada bloco $i$ corresponde um nó e a cada nó é atribuído um “peso” bi que representa o valor individual do bloco. Dados os valores dos blocos e os arcos estruturais, o algoritmo começa a construir um grafo a partir da base da explotação. Esse grafo assemelha-se a uma árvore com diversos ramos. Os ramos são denominados fortes, se o valor total dos blocos incluídos no ramo é positivo; ou fracos, em caso contrário. Os ramos vão sendo criados a partir do fundo da cava, unindo-se ou separando-se, segundo as características dos arcos estruturais, até atingirem a superfície e definindo o contorno ótimo da cava. Isto quer dizer que o fluxo máximo possível seria a soma dos valores de todos os blocos de minério, mas não é possível a retirada somente de blocos de minério. A retirada dos blocos de minério é restringida por condicionantes, como a estabilidade geral dos taludes, e, também, a seqüência dessa retirada deve ser obedecida.

Do ponto de vista da Engenharia de Minas, fechamento é simplesmente um subgrafo T, composto por nós e ramos, que gere uma cava tecnologicamente possível. Cada uma dessas cavas possíveis (subgrafos) tem um peso total associado (valor). O grande desafio para o engenheiro de minas é encontrar aquela cava única (subgrafo), entre as diversas possíveis, que conduza ao peso máximo ou ao valor máximo. Esse ajuste é chamado fechamento máximo em $G$. Formalmente, o problema de fechamento máximo de um grafo e o problema de abertura de cavas de minas são o mesmo. Do ponto de vista da Engenharia de Minas, o fechamento máximo corresponde ao fechamento de um dado conjunto de blocos que conduza à maior soma possível, considerando o peso dos blocos individuais, entre todas as alternativas tecnicamente possíveis (Hustrulid \& Kuchta, 1995).

\section{Discussão e exemplo}

O uso da programação dinâmica para o projeto de cavas de mineração foi discutido pela primeira vez por Lerchs e Grossmann (1965). O problema da otimi- zação de uma cava final (a duas ou três dimensões) não tem, na prática, uma solução analítica fácil. Tal dificuldade tem resultado no surgimento de diversos algoritmos heurísticos e variados métodos de resolução. A seguir apresentam-se os procedimentos básicos do método manual de otimização aplicando o algoritmo de Lerchs e Grossmann a uma jazida discretizada em blocos tecnológicos de lavra. A Figura 1 mostra uma seção vertical de um modelo de blocos com os valores econômicos dos blocos, $\mathrm{m}_{\mathrm{ij}}$. Os passos fundamentais do algoritmo são mostrados relacionando-se as equações pertinentes aos blocos de lavra e exemplificando com ilustrações e dados numéricos.

1) Uma vez discretizada a jazida em blocos tecnológicos de lavra apropriadamente avaliados, para cada bloco $(i, j)$, define-se a quantidade $\mathrm{m}_{\mathrm{ij}}=\mathrm{v}_{\mathrm{ij}}-\mathrm{c}_{\mathrm{ij}}$. $\mathrm{Na}$ verdade, trata-se do benefício de cada bloco, ou seja, receitas (v) menos custos (c).

2) Calcula-se o valor $M_{i j}$ acumulado para cada coluna considerada, ou seja:

$$
\mathrm{M}_{\mathrm{ij}}=\sum_{\mathrm{k}=1}^{\mathrm{i}} \mathrm{m}_{\mathrm{kj}}
$$

3) Procura-se o caminho ótimo que represente o contorno da cava para a seção considerada, sendo que, na procura desse caminho, usam-se os procedimentos e as relações abaixo:

$\mathrm{P}_{\mathrm{oj}}=0 \rightarrow$ adiciona-se uma primeira linha ao conjunto de blocos, sendo esta composta apenas por zeros.

$\mathrm{P}_{\mathrm{ij}}=\mathrm{M}_{\mathrm{ij}}+\max _{\mathrm{K}}\left\{\left(\mathrm{P}_{\mathrm{i}+\mathrm{k}, \mathrm{j}-1}\right)\right\} \mathrm{com}$

$\mathrm{k}=-1,0,+1$ no caso do talude $1: 1\left(45^{\circ}\right)$.

$\mathrm{P}_{\text {max }}=\max _{\mathrm{K}} \mathrm{P}_{\mathrm{ik} \text {. }}$

A otimização será, assim, garantida, sendo preciso notar que:

i) $\mathrm{P}_{\mathrm{ij}}$ representa a contribuição máxima possível das colunas 1 a j, para qualquer pit viável que contenha o elemento $(\mathrm{i}, \mathrm{j})$.

ii) Caso o valor máximo de $P$, na primeira linha, seja positivo, então a cava ótima é obtida seguindo-se os arcos da direita para esquerda. 
Frederico Augusto Rosa do Carmo et al.

iii) Com pequenos ajustes manuais é possível obter a cava final ótima a 3D.

Pode-se acompanhar um exemplo de otimização de uma seção bidimensional pela Figura 1. Consideraram-se os valores monetários de (-4) unidades para blocos de estéril e de 12 unidades para blocos de minério.

A otimização é iniciada fazendo-se a soma dos valores em cada uma das colunas. Exemplificando, para o bloco da linha 4 e coluna $7\left(\mathrm{M}_{47}\right)$, tem-se:

$$
\mathrm{M}_{47}=\sum_{\mathrm{k}=1}^{4} \mathrm{~m}_{\mathrm{k} 7}=\mathrm{m}_{17}+\mathrm{m}_{27}+\mathrm{m}_{37}+\mathrm{m}_{47}=12+12+12+12=48
$$

Uma nova seção, então, é formada, com os valores de $\mathrm{M}_{\mathrm{ij}}$, podendo ser vista na Figura 1c.

O próximo passo é escolher o bloco à esquerda de máximo valor, que será somado com o bloco original, obtendo-se o $\mathrm{P}_{\mathrm{ij}}$.

O processo de otimização está condicionado, logicamente, à escolha dos ângulos de talude da cava. Mudanças nos ângulos de talude requerem modificações no número de combinações para seleção dos blocos. Exemplificando, para o caso em que a relação vertical/horizontal seja de $1: 1$, com o "caminho" a ser percorrido da esquerda para a direita, tem-se a seguinte relação:

$$
P_{i j}=M_{i j}+\operatorname{Max}\left\{\begin{array}{l}
P_{i-1, j-1} \\
P_{i, j-1} \\
P_{i+1, j-1}
\end{array}\right.
$$

Sendo os valores de P, M e $\mathrm{M}_{\mathrm{ij}}$ definidos como anteriormente.

O uso do ângulo de talude de $45^{\circ}$ (1:1) é uma simplificação. É evidente que o(s) ângulo(s) de talude será(ão) determinado(s) por condicionamentos geomecânicos e de projeto.

O modelo de programação dinâmica mais completo e que leva em consideração múltiplos ângulos de talude pode ser representado pelas seguintes relações:

$$
\begin{aligned}
& \mathrm{M}_{\mathrm{ij}}=\sum_{\mathrm{n}=0}^{\mathrm{i}} \mathrm{m}_{\mathrm{nj}} \\
& \mathrm{P}_{\mathrm{ij}}=\mathrm{M}_{\mathrm{ij}}+\operatorname{Max}\left\{\mathrm{P}_{\mathrm{i}+\mathrm{r}, \mathrm{j}-1}\right\} \\
& \text { Com } \mathrm{j}=1,2, \ldots \text { e } \mathrm{r}=-n a,-n a+1, \ldots, n b
\end{aligned}
$$

Onde:

\begin{tabular}{|c|c|c|c|c|c|c|c|c|c|c|c|}
\hline-4 & -4 & 12 & 12 & $\begin{array}{l}12 \\
\end{array}$ & -4 & -4 & -4 & -4 & -4 & -4 & -4 \\
\hline-4 & -4 & -4 & 12 & 12 & 12 & -4 & -4 & -4 & -4 & -4 & -4 \\
\hline-4 & -4 & -4 & 412 & 12 & 12 & 12. -4 & -4 & -4 & -4 & -4 & -4 \\
\hline-4 & -4 & -4 & $-41^{12}$ & 12 & 12 & 12 & -4 & -4 & -4 & -4 & -4 \\
\hline-4 & -4 & -4 & -4 & $\sqrt{4} 12$ & 12 & 12 & -4 & -4 & -4 & -4 & -4 \\
\hline-4 & -4 & -4 & -4 & -4 & 12 & 12 & 12 & -4 & -4 & -4 & -4 \\
\hline-4 & -4 & -4 & -4 & -4 & 12 & 12 & 12 & -4 & -4 & -4 & -4 \\
\hline-4 & -4 & -4 & -4 & -4 & $-4 \sqrt{12}$ & 12 & 12 & 124 & -4 & -4 & -4 \\
\hline-4 & -4 & -4 & & -4 & -4 & $\sqrt{12}$ & 12 & 12 & -4 & -4 & -4 \\
\hline
\end{tabular}

$\mathrm{M}_{\mathrm{ij}}, \mathrm{m}_{\mathrm{ij}}$ e $\mathrm{P}_{\mathrm{ij}}$ como foram definidos anteriormente.

$\mathrm{r}=$ limite do talude da coluna vizinha.

na, $\mathrm{nb}=$ O número máximo de blocos acima e abaixo do bloco (i,j) que serão lavrados juntos. a) Modelo de blocos com a delimitação do corpo de minério.

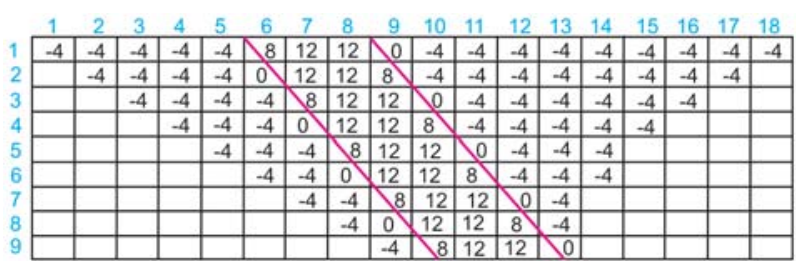

b) Modelo de blocos com os valores para a otimização.

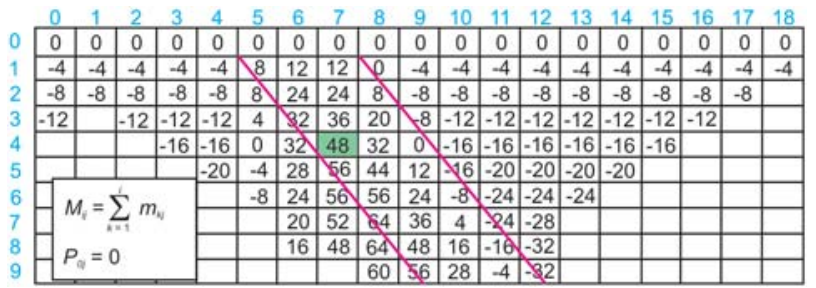

c) Cava mostrando os valores acumulados $M_{i j}$.

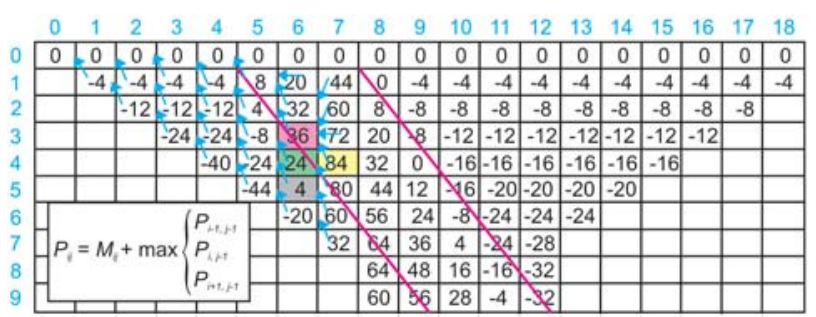

d) Escolha do máximo valor $P_{i j}$.

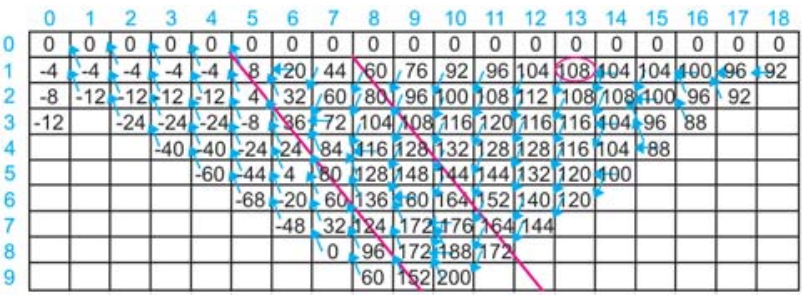

e) Procura do maior $P_{1 k}$.

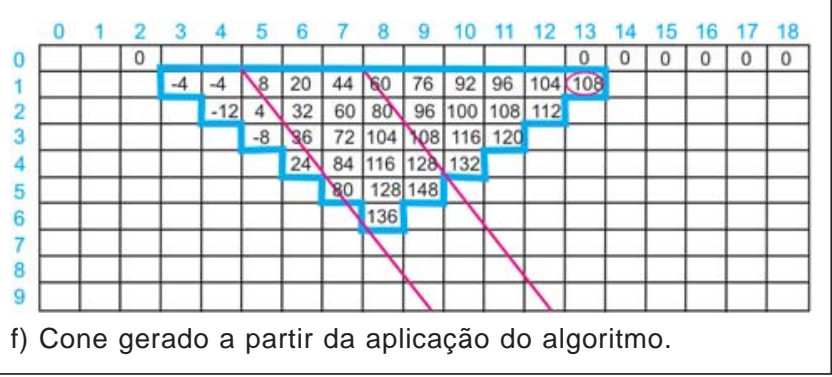

Figura 1 - Exemplo de aplicação do método manual de otimização aplicando o algoritmo de Lerchs e Grossmann a uma jazida discretizada em blocos tecnológicos de lavra. Fonte: Modificado de Lerchs e Grossmann (1965). 
Para se obter a cava tridimensional, a partir dos limites das seções bidimensionais, obtidas pela fórmula de recorrência antes apresentada, é necessária a suavização dos limites da cava. Considerando que os limites ótimos, nas seções, tenham sido alcançados, a prática de suavização é, então, empregada para adequar as seções ao modelo de cava tridimensional. Johnson e Mickle (1970) e Johnson e Sharp (1971) desenvolveram uma “extensão" ao algoritmo de programação dinâmica bidimensional de Lerchs e Grossman, a qual é conhecida como a primeira tentativa de resolver o problema de suavização dos limites da cava final. Essa extensão, embora freqüentemente mencionada como um algoritmo de programação dinâmica tridimensional completo, é intitulada com mais precisão como um algoritmo 21ำ D.

As vantagens da "extensão" de Johnson para o problema dos limites da cava final são, principalmente, a facilidade e velocidade de implementação em um computador e sua capacidade para, pelo menos parcialmente, gerar resultados tridimensionais. A desvantagem principal é que a solução obtida pode não ser fisicamente possível (praticável). Embora a extensão de Johnson não tenha sido muito usada, ela pode ser extremamente útil para a modificação de um algoritmo de contorno final. O algoritmo da extensão de Johnson é muito parecido com o algoritmo de programação dinâmica 2D original, porém apresenta a vantagem de possibilitar interações perpendiculares à direção de corte transversal. A “extensão” de Johnson e Sharp (1971) é, na verdade, uma aplicação sistemática do algoritmo bidimensional, antes apresentado. Primeiramente, o algoritmo é aplicado a todas as seções transversais e, no final, o mesmo algoritmo é aplicado a seções que façam um ângulo reto com as seções originais.

$\mathrm{O}$ algoritmo de contorno que usa a aproximação de programação dinâmica $2 \frac{1}{2} \mathrm{D}$ segue os mesmos passos do algoritmo de contorno iterativo, com a exceção do passo que considera a união das cavas das seções transversais. A “extensão” de Johnson é, com efeito, uma aplicação sistemática da fórmula de recorrência de Lerchs e Grossmann (1965). Em adição à fórmula de recorrência de Lerchs e Grossmann, a notação Siq é usada:

$\mathrm{S}_{\mathrm{iq}}=\sum_{\mathrm{ij}} \mathrm{M}_{\mathrm{ij}}$

onde $\mathrm{S}_{\mathrm{iq}}$ é a soma dos valores econômicos dos blocos do contorno ótimo da cava em uma dada seção; $M_{\mathrm{ij}}$ já foi definido anteriormente.

Duas observações fundamentais devem ser feitas em relação aos algoritmos comentados:

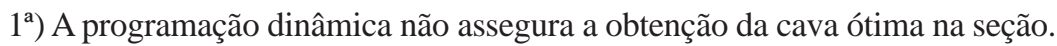

$2^{\mathrm{a}}$ ) A técnica dos cones flutuantes a três dimensões possibilita, automaticamente, a suavização do contorno da cava.

A técnica do caminho dinâmico pode ser, assim, resumida:

$1^{\circ}$ ) Aplicar a formula de recorrência, em seqüência, através das diversas seções, ao mesmo tempo em que se muda a direção de análise através das seções, quando da mudança de uma seção para a seguinte.

$2^{\circ}$ ) No final do procedimento e ao analisarem-se todos os blocos de todas as seções consecutivas, se formará um “caminho” ao longo do qual os cones mínimos de remoção do material deverão ser construídos.

$3^{\circ}$ ) A união dos cones mínimos de remoção ao longo do caminho dinâmico definirá os limites da cava final.

Koesnigsberg (1982) desenvolveu um algoritmo que é considerado, realmente, de programação dinâmica tridimensional, para o cálculo do limite da cava final (Wright, 1990). Esse algoritmo usa um otimizador, que é tido como eficaz e rápido, que atua sobre um conjunto fixo de suposições ou restrições. Como qualquer algoritmo de programação dinâmica, o algoritmo de Koesnigsberg é também cíclico e possui um grau de conformidade própria muito elevado. Para exemplificar, deve-se atentar ao fato de que os ângulos de talude da cava, em seus diversos setores, devem ser consistentes entre si e devem relacionar-se à geometria dos blocos.

Diferentemente do caso bidimensional, proposto por Lerchs e Grossmann, que considera como vizinhos próximos apenas os blocos da coluna na direção anterior ao bloco em análise, no caso tridimensional os blocos vizinhos a considerar correspondem a quatro colunas, segundo a fórmula de recorrência a seguir, as quais podem exercer influência no caminho dinâmico a ser percorrido pelo algoritmo:

$$
P_{i j k}=M_{i j k}+\operatorname{Max}\left\{\begin{array}{l}
P_{S i, j-1, k} \\
-P_{S B S(S i), j-1, k-1}+P_{B S i, j-1, k-1} \\
-P_{S(B S i), j, k-1}+P_{S B S i, j, k-1} \\
-P_{S(S B S i), j+1, k-1}+P_{S S B S i, j+1, k-1}
\end{array}\right.
$$

$\mathrm{S}_{\mathrm{i}}$ na coluna $(\mathrm{j}-1, \mathrm{k})=$ ao lado de $\mathrm{i}$.

BS $_{\mathrm{i}}$ na coluna $(\mathrm{j}-1, \mathrm{k}-1)$ = atrás do lado de $\mathrm{i}$.

$\mathrm{SBS}_{\mathrm{i}}$ na coluna $(\mathrm{j}, \mathrm{k}-1)$ = do lado de trás do lado de $\mathrm{i}$.

$\operatorname{SSBS}_{\mathrm{i}}$ na coluna $(\mathrm{j}+1, \mathrm{k}-1)=$ do lado do lado de trás do lado i.

Onde:

$\mathrm{P}_{\mathrm{ijk}}=$ Valor ótimo da cava considerando que o bloco $\mathrm{b}_{\mathrm{ijk}}$ é o último bloco a ser analisado. 
$\mathrm{M}_{\mathrm{ijk}}=$ valor $\mathrm{M}_{\mathrm{ij}}$ acumulado para cada coluna considerada.

$\mathrm{P}_{\mathrm{ijk}}=$ Valores dos vizinhos do bloco (i,j,k).

O problema de programação dinâmica tridimensional é equivalente ao problema de programação linear, cujo objetivo da função $\mathrm{P}_{\mathrm{ijk}}$ é definido por $\mathrm{P}_{\mathrm{ijk}}=\max \sum \mathrm{m}_{\mathrm{i} \mathrm{j}^{\prime} \mathrm{k}^{\prime}}, \mathrm{X}_{\mathrm{i} \mathrm{j}^{\prime} \mathrm{j}^{\prime} \text { '. }}$

\section{Comentários finais}

Existe uma dificuldade, comum a todos os algoritmos de otimização econômica de cavas finais de mineração. Essa dificuldade refere-se ao fato de os limites finais da cava não poderem ser determinados até que os valores econômicos dos blocos sejam conhecidos com maior precisão. Entretanto os valores econômicos dos blocos não podem ser conhecidos com precisão, até que a seqüência de lavra seja especificada. Por sua vez, a seqüência de lavra, eventualmente, não poderá ser especificada, até que os limites da cava sejam definidos. Trata-se de um problema recorrente que precisa ser bem equacionado.

Otimização é uma palavra que vem sendo gradualmente aceita para descrever o conjunto de técnicas que introduzem modelos matemáticos analíticos nas atividades de planejamento de mina. Entretanto o significado de otimizar, ou melhorar, é relativo. É impossível obterse unanimidade sobre o melhor plano de exaustão de uma dada mina. Para isto seria necessário o consenso de opiniões entre profissionais de diversas áreas. Em termos da modelagem da mina, a maior atenção tem-se concentrado em delinear o limite final da cava, porque este tem demonstrado ser o problema menos complexo. Freqüentemente, o problema mais crítico a ser resolvido corresponde ao seqüênciamento da lavra, ou seja, como programar a produção em relação ao tempo (Carmo \& Curi, 2001).

Um critério comumente adotado no planejamento de mina é o critério do valor presente líquido (VPL) do fluxo de caixa. Entretanto mesmo esse critério pode ser demasiadamente intuitivo dependendo da situação.
A pergunta clássica que deve ser feita é: como lidar com esse grupo de incertezas? Buscando-se atingir a otimização, têm sido propostos modelos onde é adotada, nos cálculos, a média ponderada de teores e dos preços. Entretanto, tais modelos geram planos de lavra que são limitados e pouco flexíveis a mudanças. Assim, é imprescindível que se contemplem, nesses modelos, as conseqüências de suposições diferentes, como, por exemplo, mudanças inesperadas de teor médio e variações econômicas (análises de sensibilidade). Tais modelos devem ser flexíveis, de tal forma que possibilitem o eventual refazer completo do plano, em função de cenários econômicos ou tecnológicos diferentes. Na prática mineira, entretanto, raramente são empreendidos tais exercícios. Isto é devido à quantidade e, de certa forma, complexidade dos trabalhos envolvidos, mesmo com todas as ferramentas computacionais disponíveis na atualidade. Para finalizar, informamos que, como resultado da pesquisa e produto desse trabalho, foi desenvolvido um programa acadêmico, em linguagem Delphi, que possibilita a otimização de cavas de mineração simplificadas, a partir dos valores econômicos dos blocos e usando os principais algoritmos de otimização. Esse programa encontra-se disponível para consulta na Coordenação do Programa de Pós-Graduação em Engenharia Mineral da UFOP e, também, nas bibliotecas da Escola de Minas da UFOP.

\section{Agradecimentos}

Os autores agradecem o apoio recebido da CAPES, que viabilizou a elaboração desse estudo, como também aos professores da área de concentração em Lavra de Minas do Programa de Pós-Graduação em Engenharia Mineral da UFOP, pelos comentários e discussões.

\section{Referências bibliográficas}

CARMO, F.A.R., CURI, A. Metodologias para o planejamento de cavas finais de minas a céu aberto otimizadas. Ouro
Preto: Universidade Federal de Ouro Preto-UFOP, 2001. (Dissertação de Mestrado).

HUSTRULID, W., KUCHTA, M. Open pit mine planning \& design. fundamentals. Colorado School of Mines, v. 1, Colorado, United States of America, 1995.

JOHNSON, T.B., MICKLE, D.G. Design of open pit - an application in uranium. Canadian Institute of Mining, special, v. 12, 1970.

JOHNSON, T.B., SHARP, R.W. Three dimensional dynamic programming method for optimal ultimate pit design. U.S. Bureau of Mines, Report of Investigation n. 7553, 1971.

KOENIGSBERG, E. The Optimum contours of an open pit mine: an application of dynamic programming. 17th Application of Computers and Operations Research in the Mineral Industry, Berkeley, EUA, p. 274-287, 1982.

LERCHS, H., GROSSMANN, L. F. Optimum design of open-pit mines. Transactions, Canadian Mining and Metallurgical Bulletin, Montreal, Canada, v. LXVIII, p.17-24, 1965.

VALENTE, J.M.G.P. Geomatemática - lições de geoestatística. Ouro Preto: Fundação Gorceix, v. 1-8, 1982.

WRIGHT, E.A. Open pit design models. Trans Tech Publications. Federal Republic of Germany. ClausthalZellerfeld. 1990.

\section{Artigo recebido em 13/09/2005 e aprovado em 14/07/2006.}

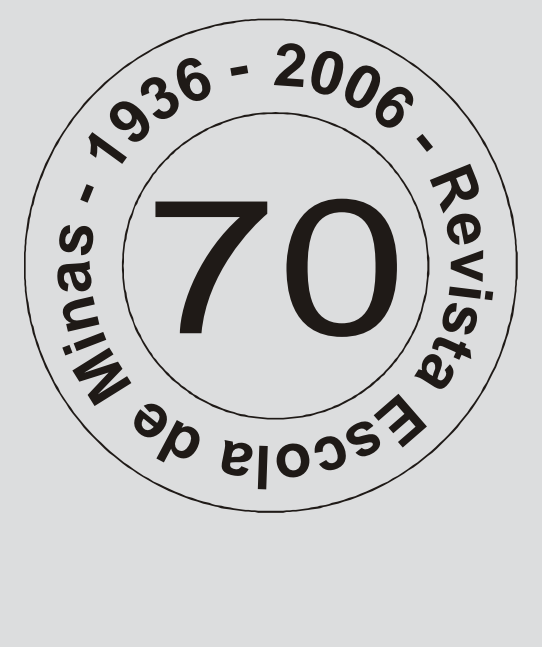

